\title{
Solution of Partial Derivative Equations of Poisson and Klein-Gordon with Neumann Conditions as a Generalized Problem of Two-Dimensional Moments
}

\author{
Maria B. Pintarelli ${ }^{1,2}$ \\ ${ }^{1}$ Department of Basic Sciences, Faculty of Engineering, National University of La Plata, La Plata, Argentina \\ ${ }^{2}$ Mathematics Department, Faculty of Exact Sciences, National University of La Plata, La Plata, Argentina \\ Email: mariabpintarelli@gmail.com
}

How to cite this paper: Pintarelli, M.B. (2020) Solution of Partial Derivative Equations of Poisson and Klein-Gordon with Neumann Conditions as a Generalized Problem of Two-Dimensional Moments. Journal of Applied Mathematics and Physics, 8, 1606-1614.

https://doi.org/10.4236/jamp.2020.88123

Received: July 22, 2020

Accepted: August 22, 2020

Published: August 25, 2020

Copyright (c) 2020 by author(s) and Scientific Research Publishing Inc.

This work is licensed under the Creative Commons Attribution International License (CC BY 4.0).

http://creativecommons.org/licenses/by/4.0/

\section{Open Access}

\begin{abstract}
It will be shown that finding solutions from the Poisson and KleinGordon equations under Neumann conditions are equivalent to solving an integral equation, which can be treated as a generalized two-dimensional moment problem over a domain that is considered rectangular. The method consists to solve the integral equation numerically using the two-dimensional inverse moments problem techniques. We illustrate the different cases with examples.
\end{abstract}

\section{Keywords}

Equation in Poisson Partial Derivatives, Klein-Gordon Equation, Integral Equations, Generalized Moment Problem

\section{Introduction}

You want to find $w(x, t)$ such that

$$
w_{x x}+w_{t t}=R(x, t) \quad \text { or } \quad w_{x x}-w_{t t}=R(x, t)
$$

with $R(x, t)$ known about a $D$ domain where

- $D=\left\{(x, t) ; a_{1}<x<b_{1}, a_{2}<t<b_{2}\right\}$

- $D=\left\{(x, t) ; a_{1}<x<b_{1}, t>a_{2}\right\}$

The underlying space is $L^{2}(D)$. Under the conditions

$$
\begin{array}{rr}
w_{x}\left(a_{1}, t\right)=k_{1}(t) & w_{x}\left(b_{1}, t\right)=k_{2}(t) \\
w_{t}\left(x, a_{2}\right)=h_{1}(t) & w_{t}\left(x, b_{2}\right)=h_{2}(t) \\
w\left(x, a_{2}\right)=s_{1}(t) & w\left(x, b_{2}\right)=s_{2}(t)
\end{array}
$$

The problem has been largely studied and solved with differen$\mathrm{t}$ methods such as the method of finite differences [1-4] to name a few. 
The objective of this work is to show that we can solve the problem using the techniques of inverse moments problem. We focus the study on the numerical approximation.

We want to present a an alternative method to solve a Poisson equation under Neumann conditions using techniques of generalized inverse moment problem, independently of other commonly used existing methods: finite difference method, Galerkin method, among many others. The interest is not to compare with the existing methods, but to present a different method to my novel criteria, and the one that I have already applied in other cases of partial differential equations under other conditions, for example the Poisson equation under Cauchy conditions or from Dirichlet. It turns out that a change in conditions implies a different approach. This is a significant change in the problem statement for its resolution.

The generalized moments problem [5-7] is to find a function $f(x)$ about a domain $\Omega \subset R^{d}$ that satisfies the sequence of equations

$$
\mu_{i}=\int_{\Omega} g_{i}(x) f(x) d x \quad i \in N
$$

where $N$ is the set of the natural numbers, $\left(g_{i}(x)\right)$ is a given sequence of functions in $L^{2}(\Omega)$ linearly independent known and the succession of real numbers $\left\{\mu_{i}\right\}_{i \in N}$ is known data. The problem of Hausdorff moments [5-7], is to find a function $f(x)$ en $(a, b)$ such that

$$
\mu_{i}=\int_{a}^{b} x^{i} f(x) d x \quad i \in N
$$

In this case $g_{i}(x)=x^{i}$ with $i$ belonging to the set $N$.

If the integration interval is $(0, \infty)$ we have the problem of Stieltjes moments; if the integration interval is $(-\infty, \infty)$ we have the problem of Hamburger moments [5-7]. The moments problem is an ill-conditioned problem in the sense that there may be no solution and if there is no continuous dependence on the given data [5-7]. There are several methods to build regularized solutions. One of them is the truncated expansion method [5]. This method is to approximate (2) with the finite moments problem

$$
\mu_{i}=\int_{\Omega} g_{i}(x) f(x) d x \quad i=1,2, \ldots, n
$$

where it is considered as approximate solution of $f(x)$ to $p_{n}(x)=$ $\sum_{i=0}^{n} \lambda_{i} \phi_{i}(x)$, and the functions $\left\{\phi_{i}(x)\right\}_{i=1, \ldots, n}$ result of orthonormalize $\left\langle g_{1}, g_{2}, \ldots, g_{n}\right\rangle$ being $\lambda_{i}$ the coefficients based on the data $\mu_{i}$. In the subspace generated by $\left\langle g_{1}, g_{2}, \ldots, g_{n}\right\rangle$ the solution is stable. If $n \in N$ is chosen in an appropriate way then the solution of (6) it approaches the solution of the original problem (2).

In the case where the data $\mu_{i}$ are inaccurate the convergence theorems should be applied and error estimates for the regularized solution (p. 19 a 30 de [5]).

\section{Resolution of the Poisson Equation}

We consider

$$
w_{x x}+w_{t t}=R(x, t)
$$

We take as an auxiliary function

$$
u(m, r, x, t)=\cos \left(\frac{m \pi}{b_{1}} x\right) e^{-r(t+1)}
$$


If the $D$ domain is bounded the conditions are:

$$
\begin{array}{r}
w_{x}\left(a_{1}, t\right)=k_{1}(t) \quad w_{x}\left(b_{1}, t\right)=k_{2}(t) \\
w_{t}\left(x, a_{2}\right)=h_{1}(x) \quad w_{t}\left(x, b_{2}\right)=h_{2}(x)
\end{array}
$$

If the region $D$ is not bounded the conditions are:

$$
w_{x}\left(a_{1}, t\right)=k_{1}(t) \quad w_{x}\left(b_{1}, t\right)=k_{2}(t) \quad w_{t}\left(x, a_{2}\right)=h_{1}(x)
$$

We define the vector field

$$
F^{*}=\left(F_{1}(w), F_{2}(w)\right)=\left(w_{x}, w_{t}\right)
$$

Since $\operatorname{div}\left(F^{*}\right)=R(x, t)$ we have to:

$$
\iint_{D} u d i v\left(F^{*}\right) d A=\iint_{D} u R(x, t) d A
$$

In addition, as $u \operatorname{div}\left(F^{*}\right)=\operatorname{div}\left(u F^{*}\right)-F^{*} \cdot \nabla u$, so

$$
\iint_{D} u d i v\left(F^{*}\right) d A=\iint_{D} \operatorname{div}\left(u F^{*}\right) d A-\iint_{D} F^{*} \cdot \nabla u d A
$$

where $\nabla u=\left(u_{x}, u_{t}\right)$.

And

$$
\iint_{D} F^{*} \cdot \nabla u d A=\iint_{D}\left(F_{1} u_{x}+F_{2} u_{t}\right) d A
$$

Integrating by parts:

$$
\begin{aligned}
& \iint_{D} F_{1} u_{x} d A=\int_{a_{2}}^{b_{2}} \int_{a_{1}}^{b_{1}} F_{1} u_{x} d x d t \\
&=\int_{a_{2}}^{b_{2}}\left(w\left(b_{1}, t\right) u_{x}\left(m, r, b_{1}, t\right)-w\left(a_{1}, t\right) u_{x}\left(m, r, a_{1}, t\right)\right) d t \\
&-\iint_{D} w u_{x x} d A \\
& u_{x}\left(m, r, b_{1}, t\right)=-e^{-r(t+1)} \operatorname{sen}\left(\frac{m \pi}{b_{1}} b_{1}\right) \frac{m \pi}{b_{1}}=0 \\
& u_{x}\left(m, r, a_{1}, t\right)=-e^{-r(t+1)} \operatorname{sen}\left(\frac{m \pi}{b_{1}} a_{1}\right) \frac{m \pi}{b_{1}} \underbrace{=}_{a_{1}=0} 0
\end{aligned}
$$

Analogously

$$
\begin{aligned}
\iint_{D} F_{2} u_{t} d A & =\int_{a_{2}}^{b_{2}} \int_{a_{1}}^{b_{1}} F_{2} u_{t} d x d t \\
& =\int_{a_{1}}^{b_{1}}\left(w\left(x, b_{2}\right) u_{t}\left(m, r, x, b_{2}\right)-w\left(x, a_{2}\right) u_{t}\left(m, r, x, a_{2}\right)\right) d x \\
& -\iint_{D} w u_{t t} d A
\end{aligned}
$$

If $m \in N$ y $a_{1}=0$ then

$$
\begin{aligned}
& \iint_{D} F^{*} \cdot \nabla u d A \\
& =\int_{a_{1}}^{b_{1}}\left(w\left(x, b_{2}\right) u_{t}\left(m, r, x, b_{2}\right)-w\left(x, a_{2}\right) u_{t}\left(m, r, x, a_{2}\right)\right) d x \\
& -\iint_{D} w\left(u_{x x}+u_{t t}\right) d A
\end{aligned}
$$


where

$$
\iint_{D} w\left(u_{x x}+u_{t t}\right) d A=\iint_{D} w u\left(-\left(\frac{m \pi}{b_{1}}\right)^{2}+(t+1)^{2}\right) d A
$$

On the other hand,

$$
\begin{aligned}
& \int_{C}\left(u F^{*}\right) \cdot n d s \\
& =-\int_{a_{1}}^{b_{1}} u\left(m, r, x, a_{2}\right) w_{t}\left(x, a_{2}\right) d x \int_{a_{1}}^{b_{1}} u\left(m, r, x, b_{2}\right) w_{t}\left(x, b_{2}\right) d x \\
& +\int_{a_{2}}^{b_{2}} u\left(m, r, b_{1}, t\right) w_{x}\left(b_{1}, t\right) d t \\
& -\int_{a_{2}}^{b_{2}} u\left(m, r, a_{1}, t\right) w_{x}\left(a_{1}, t\right) d t=G(m, r) \\
& \quad \therefore \iint_{D} u R(x, t) d A=G(m, r) \\
& \quad-\int_{a_{1}}^{b_{1}}\left(w\left(x, b_{2}\right) u_{t}\left(m, r, x, b_{2}\right)-w\left(x, a_{2}\right) u_{t}\left(m, r, x, a_{2}\right)\right) d x \\
& \quad+\iint_{D} w u\left(-\left(\frac{m \pi}{b_{1}}\right)^{2}+(r+1)^{2}\right) d A \\
& \therefore \iint_{D} w u\left(-\left(\frac{m \pi}{b_{1}}\right)^{2}+(r+1)^{2}\right) d A \\
& =-\int_{a_{1}}^{b_{1}}\left(w\left(x, b_{2}\right) u_{t}\left(m, r, x, b_{2}\right)-w\left(x, a_{2}\right) u_{t}\left(m, r, x, a_{2}\right)\right) d x \\
& -G(m, r)+\iint_{D} u R(x, t) d A=\varphi(m, r) \\
& \quad \therefore \iint_{D} w u d A=\frac{\varphi(m, r)}{\left(-\left(\frac{m \pi}{b_{1}}\right)^{2}+(r+1)^{2}\right)}
\end{aligned}
$$

If the equation is from Klein-Gordon, it is taken

$$
\begin{gathered}
F^{*}=\left(F_{1}(w), F_{2}(w)\right)=\left(w_{x},-w_{t}\right) \\
\therefore \iint_{D} w u d A=\frac{\varphi^{*}(m, r)}{\left(-\left(\frac{m \pi}{b_{1}}\right)^{2}-(t+1)^{2}\right)}
\end{gathered}
$$

where, in $\varphi^{*}(m, r)$ we have to $G(m, r)$ is different.

To solve this integral equation we give integer values to $m$ and $r$ :

$$
m=0,1,2, \ldots n_{1}-1 ; \quad r=1,2, \ldots, n_{2}
$$

then

$$
\iint_{D} w(x, t) H_{m r}(x, t) d A=\frac{\varphi(m, r)}{\left(-\left(\frac{m \pi}{b_{1}}\right)^{2}+(t+1)^{2}\right)}=\mu_{m r}
$$


We interpret (8) as a moments problem of two-dimensional generalized. $p_{n}(x, t)$ is the numerical approximation with the truncated expansion method for $w(x, t)$ with

$$
\begin{aligned}
n & =n_{1} \cdot n_{2} \\
H_{m r}(x, t)=u(m, r, x, t) \quad m & =0,1,2, \ldots n_{1}-1 ; \quad r=1,2, \ldots, n_{2}
\end{aligned}
$$

\section{Solution of the Generalized Moments Problem}

We can apply the detailed truncated expansion method in [7] and generalized in [8] and [9] to find an approximation $p_{n}(x, t)$ for the corresponding finite problem with $i=0,1,2, \ldots, n$, where $n$ is the number of moments $\mu_{i}$. We consider the basis $\phi_{i}(x, t) i=0,1,2, \ldots, n$ obtained by applying the Gram-Schmidt orthonormalization process on $H_{i}(x, t) i=0,1,2, \ldots, n$. We approximate the solution $w(x, t)$ with [7] and generalized in [8] y [9]:

$$
p_{n}(x, t)=\sum_{i=0}^{n} \lambda_{i} \phi_{i}(x, t) \quad \text { donde } \quad \lambda_{i}=\sum_{j=0}^{i} C_{i j} \mu_{j} \quad i=0,1,2, \ldots, n
$$

And the coefficients $C_{i j}$ verify

$$
\begin{array}{r}
C_{i j}=\left(\sum_{k=j}^{i-1}(-1) \frac{\left\langle H_{i}(x, t) \mid \phi_{k}(x, t)\right\rangle}{\left\|\phi_{k}(x, t)\right\|^{2}} C_{k j}\right) . \\
\left\|\phi_{i}(x, t)\right\|^{-1} 1<i \leq n ; 1 \leq j<i
\end{array}
$$

The terms of the diagonal are

$$
C_{i i}=\left\|\phi_{i}(x, t)\right\|^{-1} \quad i=0,1, \ldots, n .
$$

The proof of the following theorem is in $[9,10]$. In [10] the demonstration is made for $b_{2}$ finite. If $b_{2}=\infty$ instead of taking the Legendre polynomials we take the Laguerre polynomials. En [11] the demonstration is made for the one-dimensional case.

This Theorem gives a measure about the accuracy of the approximation.

\subsection{Theorem}

Let $\left\{\mu_{i}\right\}_{i=0}^{n}$ be a set of real numbers and suppose that $f(x, t) \in$ $L^{2}\left(\left(a_{1}, b_{1}\right) \times\left(a_{2}, b_{2}\right)\right)$ for two positive numbers $\varepsilon$ and $M$ verify:

$$
\begin{gathered}
\sum_{i=0}^{n}\left|\int_{a_{2}}^{b_{2}} \int_{a_{1}}^{b_{1}} H_{i}(x, t) f(x, t) d x d t-\mu_{i}\right|^{2} \leq \varepsilon^{2} \\
\int_{a_{2}}^{b_{2}} \int_{a_{1}}^{b_{1}}\left(\left(b_{1}-a_{1}\right)^{2} f_{x}^{2}+\left(b_{2}-a_{2}\right)^{2} f_{t}^{2}\right) d x d t \leq M^{2}
\end{gathered}
$$

then

$$
\begin{aligned}
& \int_{a_{2}}^{b_{2}} \int_{a_{1}}^{b_{1}}|f(x, t)|^{2} d x d t \\
& \leq \min _{i}\left\{\left\|C C^{T}\right\| \varepsilon^{2}+\frac{M^{2}}{8(i+1)^{2}} ; i=0,1, \ldots, n\right\}
\end{aligned}
$$


where $C$ it is a triangular matrix with elements $C_{i j}(1<i \leq n ; 1 \leq$ $j<i$ ) and

$$
\int_{a_{2}}^{b_{2}} \int_{a_{1}}^{b_{1}}\left|p_{n}(x, t)-f(x, t)\right|^{2} d x d t \leq\left\|C C^{T}\right\| \varepsilon^{2}+\frac{M^{2}}{8(n+1)^{2}}
$$

If $b_{2}$ it is not finite then (9) change by

$$
\int_{a_{2}}^{b_{2}} \int_{a_{1}}^{b_{1}}\left(x f_{x}^{2}+t f_{t}^{2}\right) d x d t \leq M^{2}
$$

And it must be fulfilled that

$$
t^{i} f(x, t) \longrightarrow 0 \quad \text { if } \quad t \longrightarrow \infty \quad \forall i \epsilon N
$$

\subsection{Numerical Examples}

\subsubsection{Example 1}

We consider the equation

$$
w_{x x}+w_{t t}=-3 \cos (2 x) \operatorname{Cosech}(\pi) \operatorname{Senh}(t) \quad \text { en } \quad(0,2) \times(0,2)
$$

whose solution is: $w(x, t)=\cos (2 x) \frac{\operatorname{senh}(t)}{\operatorname{senh}(\pi)}$.

We take $n=9$ moments and is approaching $w(x, t)$ where the accuracy is

$$
\int_{0}^{2} \int_{0}^{2}\left(p_{9}(x, t)-w(x, t)\right)^{2} d t d x=0.0415341
$$

In Figure 1 the graphics of: $p_{9}(x, t)$ (color magenta) $y w(x, t)$ (color celeste) are superimposed.

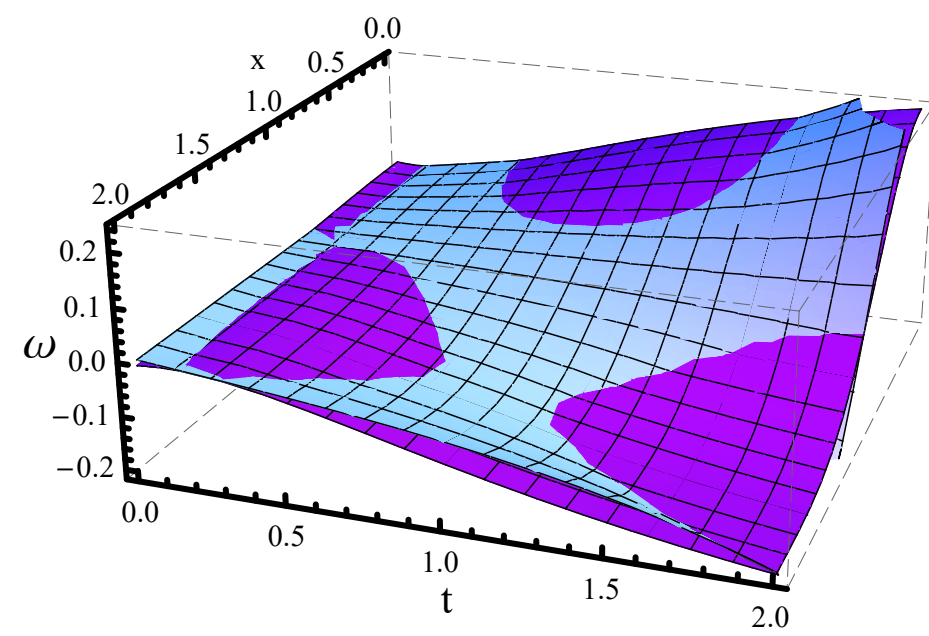

Figure 1. $p_{9}(x, t)$ and $w(x, t)$ for example 1.

\subsubsection{Example 2}

We consider the equation

$$
w_{x x}+w_{t t}=0 \quad \text { en } \quad(0,1) \times(0, \infty)
$$


whose solution is: $\operatorname{sen}(x) e^{-t}$.

We take $n=9$ moments and is approaching $w(x, t)$ where the accuracy is

$$
\int_{0}^{1} \int_{0}^{\infty}\left(p_{9}(x, t)-w(x, t)\right)^{2} d t d x=0.0193919
$$

In Figure 2 the graphics of: $p_{9}(x, t)$ (color magenta) $y w(x, t)$ (color celeste) are superimposed.

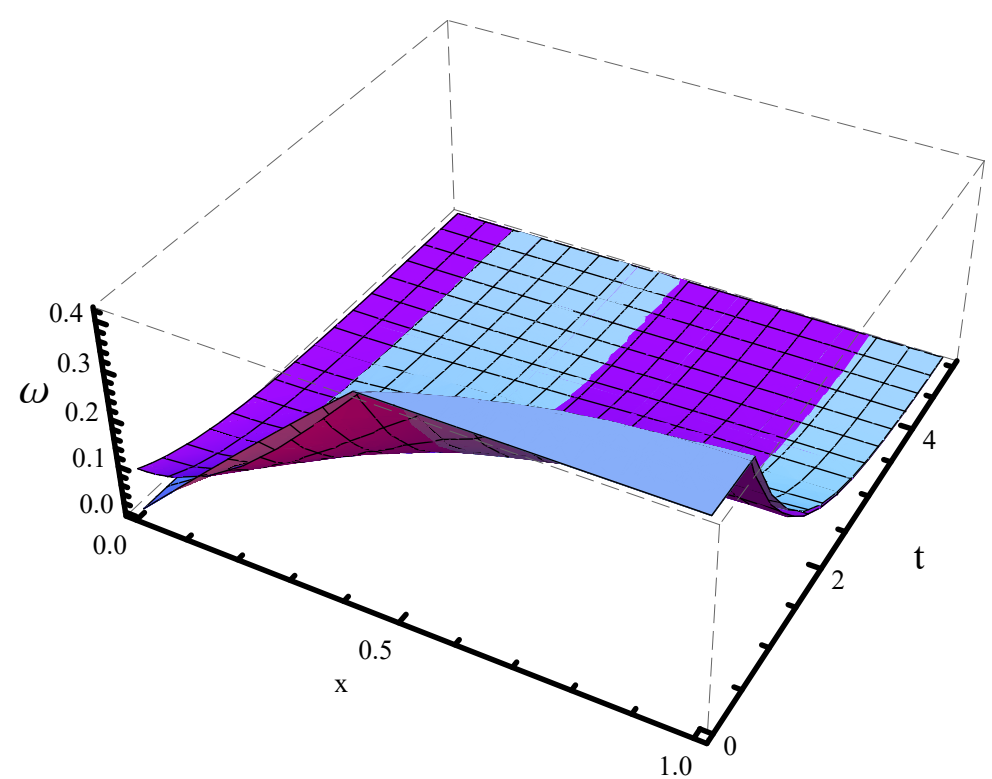

Figure 2. $p_{9}(x, t)$ and $w(x, t)$ for example 2 .

\subsubsection{Example 3}

We consider the equation

$$
w_{x x}-w_{t t}=-8 e^{-3 t+x} \quad \text { en } \quad(0,1) \times(0,2)
$$

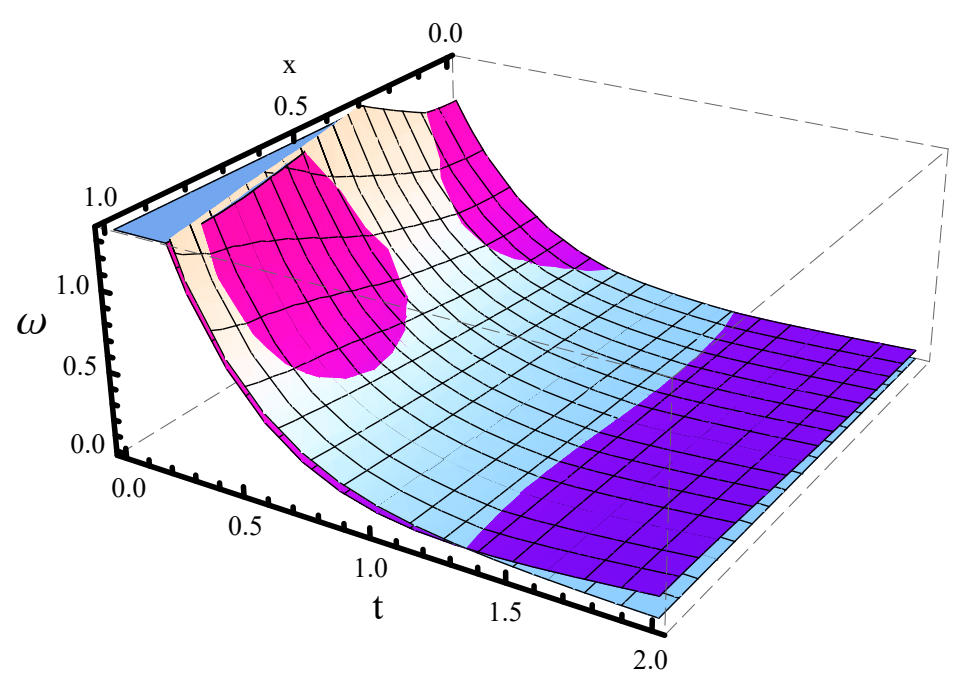

Figure 3. $p_{9}(x, t)$ and $w(x, t)$ for example 3. 
whose solution is: $e^{x-3 t}$.

We take $n=9$ moments and is approaching $w(x, t)$ where the accuracy is

$$
\int_{0}^{1} \int_{0}^{2}\left(p_{9}(x, t)-w(x, t)\right)^{2} d t d x=0.0552525
$$

In Figure 3: $p_{9}(x, t)$ (color magenta) $y w(x, t)$ (color celeste) are superimposed.

\section{Conclusions}

An equation in partial Poisson derivatives of the form $w_{x x}+w_{t t}=$ $R(x, t)$ or from Klein-Gordon $w_{x x}-w_{t t}=R(x, t)$ where the unknown function $w(x, t)$ is defined in $D=\left(0, b_{1}\right) \times\left(a_{2}, b_{2}\right)$ or $D=\left(0, b_{1}\right) \times$ $\left(a_{2}, \infty\right)$ under Neumann's conditions can be solved numerically by applying inverse moment problem techniques.

1. First the partial derivatives equation is written as an integral equation

$$
\therefore \iint_{D} w u d A=\frac{\varphi(m, r)}{\left(-\left(\frac{m \pi}{b_{1}}\right)^{2}+(r+1)^{2}\right)}
$$

2. To solve this integral equation we give integer values to $m$ and $r$ :

$$
m=0,1,2, \ldots n_{1}-1 ; \quad r=1,2, \ldots, n_{2}
$$

then

$$
\iint_{D} w(x, t) H_{m r}(x, t) d A=\frac{\varphi(m, r)}{\left(-\left(\frac{m \pi}{b_{1}}\right)^{2}+(t+1)^{2}\right)}=\mu_{m r}
$$

We have a problem of two-dimensional generalized moments. $p_{n}(x, t)$ is a numerical approximation with the truncated expansion method for $w(x, t)$, where $n=n_{1} \cdot n_{2} H_{m r}(x, t)=$ $u(m, r, x, t) m=0,1,2, \ldots n_{1} ; r=1,2, \ldots, n_{2}$.

\section{Acknowledgements}

I appreciate the support of the Faculty of Engineering and the Faculty of Exact Sciences of the National University of La Plata, Argentina.

\section{Conflicts of Interest}

The author declares no conflicts of interest regarding the publication of this paper.

\section{References}

[1] Lahti, P., Maly, L. and Shanmugalingam, N. (2018) An Analog of the Neumann Problem for the 1-Laplace Equation in the Metric Setting: Existence, Boundary Regularity, and Stability. Analysis and Geometry in Metric Spaces, 6, 1-31. https://doi.org/10.1515/agms-2018-0001 
[2] Gueye, S.B., Talla, K. and Mbow, C. (2014) Solution of 1D Poisson Equation with Neumann-Dirichlet and Dirichlet-Neumann Boundary Conditions, Using the Finite Difference Method. Journal of Electromagnetic Analysis and Applications, 6, 309-318. https://doi.org/10.4236/jemaa.2014.610031

[3] Rieger, H., Projahn, U. and Beer, H. (2007) Fast Iterative Solution of Poisson Equation with Neumann Boundary Conditions in Nonorthogonal Curvilinear Coordinate Systems by a Multiple Grid Method. Numerical Heat Transfer, 6, 1-15.

https://doi.org/10.1080/01495728308963070

[4] Ma, Z.H., Chew, W.C. and Jiang, L.J. (2013) A Novel Fast Solver for Poissons Equation with Neumann Boundary Condition. Progress in Electromagnetics Research, 136, 195-209. https://doi.org/10.2528/PIER12112010

[5] Ang, D.D., Gorenflo, R., Le, V.K. and Trong, D.D. (2002) Moment Theory and Some Inverse Problems in Potential Theory and Heat Conduction. In: Lectures Notes in Mathematics, SpringerVerlag, Berlin.

[6] Shohat, J.A. and Tamarkin, J.D. (1943) The Problem of Moments. In: Mathematical Surveys and Monographs, Vol. 1, American Mathematical Society, Providence, RI.

https://doi.org/10.1090/surv/001

[7] Talenti, G. (1987) Recovering a Function from a Finite Number of Moments. Inverse Problems, 3, 501-517. https://doi.org/10.1088/0266-5611/3/3/016

[8] Pintarelli, M.B. and Vericat, F. (2008) Stability Theorem and Inversion Algorithm for a Generalize Moment Problem. Far East Journal of Mathematical Sciences, 30, 253-274 .

[9] Pintarelli, M.B. and Vericat, F. (2011) Bi-Dimensional Inverse Moment Problems. Far East Journal of Mathematical Sciences, $\mathbf{5 4}, 1-23$.

[10] Pintarelli, M.B. (2015) Linear Partial Differential Equations of First Order as Bi-Dimensional Inverse Moment Problem. Applied Mathematics, 6, 979-989. https://doi.org/10.4236/am.2015.66090

[11] Pintarelli, M.B. (2016) Parabolic Partial Differential Equations as Inverse Moments Problem. Applied Mathematics, 7, 77-99. https://doi.org/10.4236/am.2016.71007 